\title{
Unveiled feather microcosm: feather microbiota of passerine birds is closely associated with host species identity and bacteriocin- producing bacteria
}

\author{
Veronika Gvoždíková Javůrková $\mathbb{C}^{1,2} \cdot$ Jakub Kreisinger ${ }^{3}$ Petr Procházka $\mathbb{1}^{2}$ • Milica Požgayováa ${ }^{2}$ \\ Kateřina Ševčíková ${ }^{4} \cdot$ Vojtěch Brlík $\mathbb{1}^{2} \cdot$ Peter Adamík ${ }^{4} \cdot$ Petr Heneberg $\mathbb{1}^{5} \cdot$ Jiří Porkert $^{6}$
}

Received: 14 May 2018 / Revised: 12 April 2019 / Accepted: 3 May 2019 / Published online: 24 May 2019

(c) The Author(s) 2019. This article is published with open access

\begin{abstract}
The functional relevance of microbiota is a key aspect for understanding host-microbiota interactions. Mammalian skin harbours a complex consortium of beneficial microorganisms known to provide health and immune-boosting advantages. As yet, however, little is known about functional microbial communities on avian feathers, including their co-evolution with the host and factors determining feather microbiota (FM) diversity. Using 16S rRNA profiling, we investigated how host species identity, phylogeny and geographic origin determine FM in free-living passerine birds. Moreover, we estimated the relative abundance of bacteriocin-producing bacteria (BPB) and keratinolytic feather damaging bacteria (FDB) and evaluated the ability of BPB to affect FM diversity and relative abundance of FDB. Host species identity was associated with feather bacterial communities more strongly than host geographic origin. FM functional properties differed in terms of estimated BPB and FDB relative abundance, with both showing interspecific variation. FM diversity was negatively associated with BPB relative abundance across species, whereas BPB and FDB relative abundance was positively correlated. This study provides the first thorough evaluation of antimicrobial peptides-producing bacterial communities inhabiting the feather integument, including their likely potential to mediate niche-competition and to be associated with functional speciesspecific feather microbiota in avian hosts.
\end{abstract}

Supplementary information The online version of this article (https:// doi.org/10.1038/s41396-019-0438-4) contains supplementary material, which is available to authorised users.

\section{Veronika Gvoždíková Javůrková}

veronika.javurkova@gmail.com

1 Faculty of Agrobiology, Food and Natural Resources, Department of Animal Science, Czech University of Life Sciences, Kamýcká 129, 16500 Prague-Suchdol, Czech Republic

2 Institute of Vertebrate Biology, Czech Academy of Sciences, Květná 8, 60365 Brno, Czech Republic

3 Faculty of Science, Department of Zoology, Charles University, Viničná 7, 12844 Prague, Czech Republic

4 Faculty of Science, Department of Zoology, Palacký University, 17. listopadu 50, 77146 Olomouc, Czech Republic

5 Third Faculty of Medicine, Charles University, Ruská 87, 10000 Prague, Czech Republic

6 Home address: Gočárova třída 542/12, 50002 Hradec Králové, Czech Republic

\section{Introduction}

Intensive microbiome studies across a range of ecosystems and animal taxa have revealed apparent co-evolution between microbes and their hosts [1,2]. As such, investigations of host-microbiota interactions could prove fundamental to our understanding of host physiology [3], immunity [4], ecology [5, 6] and evolution of life history traits [7]. The past decade has witnessed extensive investigation into the microbiota of mammals and other vertebrates [8]. Recently, however, there has been increasing interest in the microbiota of birds. Despite a dominance of studies focused on commercially exploited species [9, 10], freeliving birds and their wild microbiota have become a pivotal theme in avian microbiome research. Most of these studies have focused on gut microbiota, with New World vultures showing a highly conserved and selective gut microbiota resulting from diet specialisation [11], co-diversification of gastrointestinal microbiota and phylogeny in passerines [12] and temporal stability and transgenerational transfer of gut microbiota in a socially living bird [13]. Aside from three 
recent studies [14-16], however, the microbiota inhabiting skin and its derivatives in free-living birds have remained largely neglected.

Recently, a number of studies have highlighted the importance of vertebrate skin microbiota. Skin microbiota have been shown to affect skin health/disease balance [17], provide antimicrobial protection to progeny [18] and even to regulate expression of innate immune genes in humans and mice $[19,20]$. In amphibians, skin microbiota communities have been found to protect anuran and caudate amphibians against chytrid fungus infection and other invading pathogens $[21,22]$. Feathers are unique keratin skin derivatives, representing an important evolutionary novelty of theropod dinosaurs and their recent descendants, birds [23]. Previous research using culture-based methods has revealed the presence of diverse microbiota inhabiting the feather surface, and has suggested links between these bacterial communities and heath- or condition-related traits in avian hosts [24-26]. To date, however, only three detailed studies using deep 16S rRNA sequencing have been published on microbiota inhabiting skin and its derivatives in free-living birds [14-16].

In the present study, we investigate feather microbiota (FM) diversity and functional properties in free-living passerine birds, with a special focus on feather damaging bacteria (FDB) and bacteriocin-producing bacteria (BPB). Up to now, the vast majority of culture-based feather microbial assemblage studies have examined FDB synthesising a wide range of keratinases capable of degrading keratinous substrates [27], including feathers [28]. FDB have been shown to impair feather structural quality in vitro [29-31], colour-signalling function [32, 33] and even host immunity [26] in free-living birds. Other studies, however, have documented no detrimental effect of FDB on feather wear quality [34, 35] or a positive synergistic effect, whereby FDB-produced keratinase amplifies the potential of preen gland secretions to eliminate specific FDB from feathers [36]. Moreover, some FDB have been documented as simultaneously producing antibiotics and bacteriocins [37]. Bacteriocins produced by BPB are ribosomally synthesised peptides either antagonising individual bacterial strains $[38,39]$, or may be non-selective targeting any kind of microorganism [40, 41]. It would appear, therefore, that FDB do not have to be only members of FM impairing host's feather integument. Alternatively, FDB able to produce bacteriocins can also act as beneficial symbionts for the host maintaining niches with nearly identical dominant strains, while out-competing individual microbial strains, or may be broadly antibiotic for a wide spectrum of microorganisms in FM including pathogens. BPB have been identified in the human and mammalian gastrointestinal tract, where they mediate niche-competition and maintain commensal community structure [42, 43]. In birds, BPB have only been detected in one species to date, the hoopoe (Upupa epops), where they were identified on both the surface and in secretions of the single bird sebaceous gland, the preen gland [44-46]. Since BPB may be transmitted from the surface of the preen gland to the feathers via preening [47], we hypothesise that the occurrence of BPB may shape and maintain host-specific FM in birds.

Here, we investigate the FM of seven free-living passerine birds using high throughput 16S rRNA sequencing and test whether feather microbiota diversity and composition is predicted by host species identity and/or its geographic origin. Furthermore, using a bioinformatics approach combining information obtained from public databases (BAGEL4, SILVA) with extensive literature mining, we estimate relative BPB and FDB abundances within FM profiles. This sort of information allowed us to investigate possible correlation between $\mathrm{BPB}$ and FDB relative abundances. Assuming that FDB primarily produce keratinases and only limited fraction of FDB can simultaneously produce bacteriocins, we may expect antagonism between BPB and FDB predicting negative correlation between BPB and FDB. Alternatively, as there might be considerable subset of FDB capable to produce bacteriocins, opposite direction of correlation is possible to be expected. Furthermore, based on ability of BPB to produce bacteriocins with broad and also specific antimicrobial potentials, we predict that there will be association between BPB and FM alpha diversity. Specifically, if we consider BPB as broad range non-selective antagonists, FM alpha diversity is expected to decrease with increasing BPB relative abundance. Alternatively, if taking into account BPB producing bacteriocins with antimicrobial activity specific to rather narrow range of bacteria, this may result in complex competition patterns keeping variety of niches not fully occupied and causing increase in FM alpha diversity with increasing BPB relative abundance.

\section{Material and methods}

\section{Ethical statement}

See Supplementary Methods.

\section{Study species and feather sample collection}

Chest contour feathers (approx. 10-15) were collected from two resident and five long-distance migratory passerines (Supplementary Table S1) at different localities within the Czech Republic (Supplementary Fig. S1). To control for the potentially confounding effect of breeding period on FM, individuals of each species were mist-netted at the beginning of their breeding season during February-April 2015. 
To avoid feather contamination caused by handling, chest contour feathers were plucked directly from each individual trapped in a mist net using microbial DNA-free forceps (i.e., the forceps tip was dipped into ethanol and passed through a flame). The feathers were immediately put into a sterile $1.8 \mathrm{~mL}$ cryotube (Deltalab, Barcelona, Spain) and kept cool until delivery to the laboratory, where the samples were subsequently stored at $-20{ }^{\circ} \mathrm{C}$ until DNA extraction.

\section{DNA extraction and 16S rRNA gene amplicon sequencing}

To assess FM, microbial genomic DNA from whole feather samples was aseptically extracted using the RTP ${ }^{\circ}$ Bacteria DNA Mini kit (STRATEC Molecular, Berlin, Germany), following Protocol 5 of the isolation kit (Isolation of bacterial DNA from tissue biopsies).

16S rRNA amplicon libraries were prepared and sequenced based on protocols already described in previous studies [12, 48]. See Supplementary Methods for full description of 16S rRNA gene amplicon sequencing and bioinformatic processing methods [49-57].

\section{Bioinformatic estimation of BPB and FDB}

To estimate BPB and FDB relative abundances within species-specific FM, all 16S rRNA sequences for BPB enlisted in the BAGEL4 database [58] (available at: http://ba gel4.molgenrug.nl/databases.php), for bacterial species with documented keratinolytic activity listed in the most recent review [27] and in Supplementary Table S2, were extracted from SILVA (version 128; [59]) and used as a reference. Next, our OTU sequences were mapped against this reference database using the UCLUST algorithm [60] with a 97\% similarity threshold corresponding approximately to specieslevel mapping. Finally, the proportion of mapped highquality reads for each sample was estimated and used as a proxy for BPB and keratinolytic FDB relative abundance. These steps were repeated separately for each of the three BPB classes producing different types of bacteriocins (i.e., Class I, Class II and Class III; see the BAGEL4 database for details) varying in structure and antimicrobial spectrum [61]. The sequence similarity threshold used for OTU sequence mapping did not affect the general conclusions of our analyses. Indeed, we observed high concordance in terms of sample-specific proportions of mapped reads at the 97 and 95\% similarity threshold (Pearson correlation: $r>0.8$ for all BPB classes and for keratinolytic FDB).

\section{Statistical analyses}

Shannon diversity indices and number of OTUs for individual samples were estimated using rarefaction-based normalised OTU tables (i.e., random sub-setting of read counts per sample was 2299 corresponding to minimal sequencing depth). Subsequently, interspecific differences in microbial diversity were analysed using Analysis of Variance (ANOVA).

Four types of dissimilarity indices, i.e., weighted and unweighted UniFrac [62], Bray-Curtis and a binary version of Jaccard dissimilarity, were used to assess differences in FM composition. Jaccard and unweighted UniFrac dissimilarity only account for OTU presence vs. absence and hence are more sensitive to FM changes driven by rare OTUs than Bray-Curtis and weighted UniFrac dissimilarity. In addition, both unweighted and weighted UniFrac dissimilarity take OTU genetic similarity into account, and hence are more sensitive to community divergence driven by phylogenetically distant bacterial taxa.

Among-sample divergence in FM composition was visualised using Non-metric Multidimensional Scaling (NMDS). We then assessed whether there was any correlation between geographic distance between sampling localities (see Supplementary Table S1 and Fig. S1) and FM dissimilarity using the Mantel test. Next, distance-based Redundancy Analyses (db-RDAs), using species identity and geographic distance as response variables, were employed to assess whether there was any interspecific divergence in FM profile composition, while accounting for clinal microbiota variation between sampling localities and vice versa. Their marginal significance (i.e., the effect of species identity controlling for the effect of spatial variation and vice versa) was subsequently tested using anova.cca. The matrix of geographic distance between sampling localities was scaled using Principal Coordinates of Neighbourhood Matrices (PCNM; [63]), the resulting PCNM score matrix being included in the db-RDA models. To prevent model overfitting, scores of the first three PCNM axes only were considered. In order to visualise the effect of geographic distance vs. interspecific variation on FM composition, we constructed a heatmap for the dominant bacterial OTUs detected in FM (i.e., represented by $>$ reads on average in at least one species) using the $\mathrm{R}$ package NMF [64].

To test for phylosymbiosis, we used the Procrustean Approach to Cophylogeny (R package PACo; [65]). Specifically, we searched for a correlation between the matrix of cophenetic phylogenetic distances of host species and matrices for microbiota composition dissimilarities, both being scaled via Principal Coordinate Analysis (PCoA) prior to PACo analyses. Cophenetic distances were calculated based on 1000 Bayesian trees randomly downloaded from http://birdtree.org/ [66]. Significance testing was based on a comparison of Procrustean sums of squares for the original dataset with the distribution of Procrustean sums of squares for random communities constructed by the PACo 
Fig. 1 Barplots showing relative abundance of 16S rRNA reads for the (a) dominant bacterial phyla and (b) classes in particular feather microbiota samples (ID) and host passerine species $(\mathrm{AA}=$ Acrocepalus arundinaceus, $\mathrm{LL}=$ Locustella luscinioides, $\mathrm{SE}=$ Sitta europaea, $\mathrm{PA}=$ Periparus ater, $\mathrm{FA}=$ Ficedula albicollis, $\mathrm{PP}=$ Phoenicurus phoenicurus, RR = Riparia riparia)

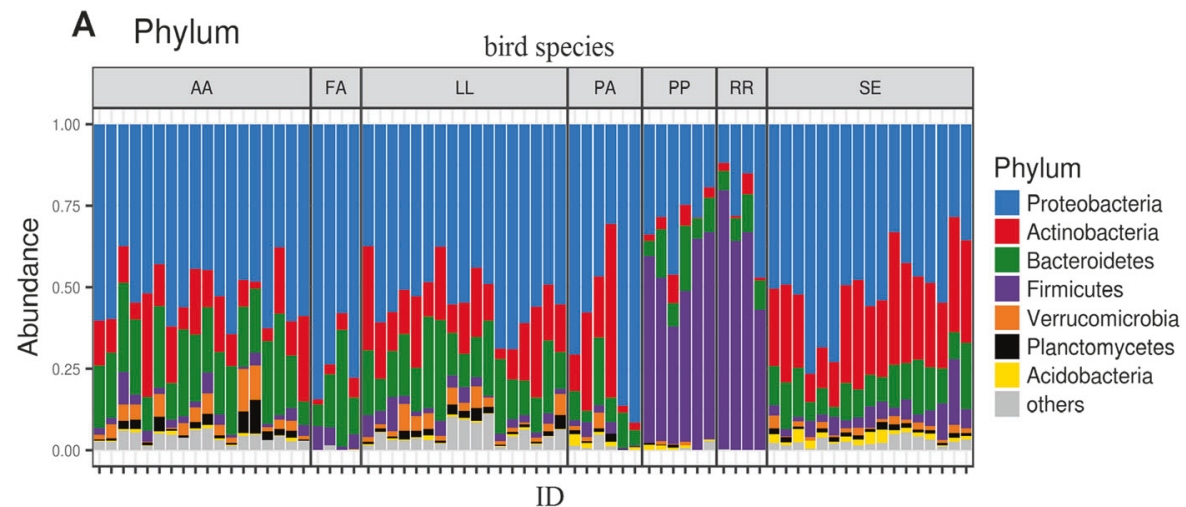

B Class

ID

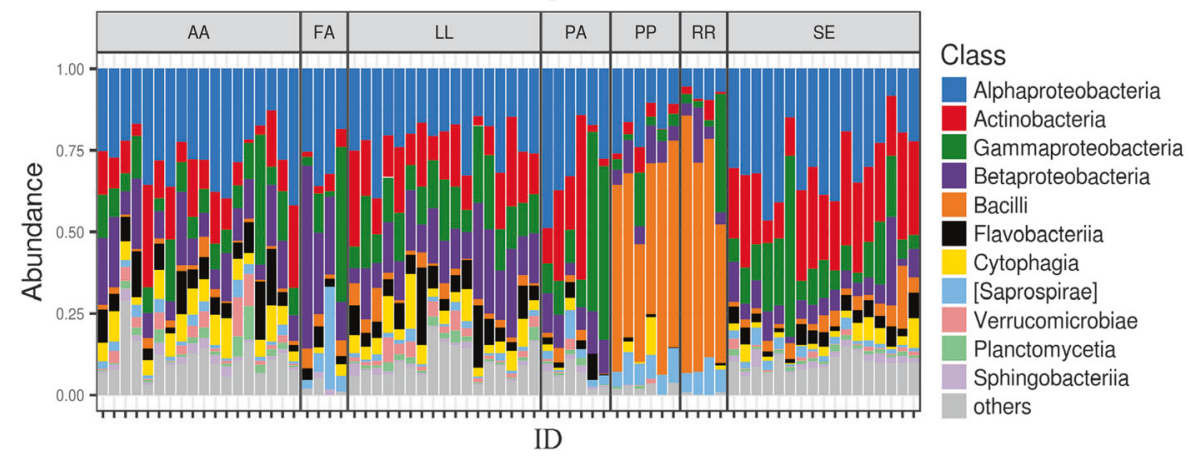

R function ( $n=10,000$ permutations). To account for the fact that the species sampled were not equally distributed between geographic localities, FM divergence partitions associated with phylogeny, geography and both of these predictors were estimated using the varpart function ( $\mathrm{R}$ package vegan) using db-RDA models that consider both the effect of phylogeny and geography. To build the $\mathrm{db}$ RDAs, dissimilarity indices scaled by PCoA were included as response variables, while sample location identity, PCNM scores for sample location distances and PCoA gradients in phylogenetic cophenetic distances were considered as potential explanatory variables. To prevent model overfitting, a specific set of explanatory variables for each $\mathrm{db}-\mathrm{RDA}$ was selected using the forward selection procedure (ordistep function in the $\mathrm{R}$ package vegan). In the results, we report the proportion of variance explained after adjusting for model complexity (i.e., adjusted $R^{2}$ ).

Finally, we used Markov Chain Monte Carlo Generalized Linear Mixed Models (R package MCMCglmm; [67]) to assess potential relationships between FM Shannon diversities and estimated relative abundances of BPB or FDB, while taking into account covariance due to shared phylogenetic ancestry. To improve model convergence, BPB and FDB relative abundances were log transformed prior to calculation. To distinguish between processes operating at the inter and intra-specific level, host-specific means of all variables were included as model predictors, along with deviations of each observation from the within- host average [68-71]. We also included the interaction between intra-specific variation and species identity to test for consistency of intra-specific effects among host species sampled. Host species identity and phylogenetic covariance were modelled as random effects.

To account for uncertainty in phylogenetic reconstruction, we conducted separate MCMC simulations for a random sample of 100 Bayesian phylogenetic trees. See Supplementary Methods for full description of MCMC simulations.

\section{Results}

\section{Taxonomic composition of feather microbiota}

The FM profiles of almost all passerine species examined, apart from the sand martin (Riparia riparia) and common redstart (Phoenicurus phoenicurus), were dominated by the phyla Proteobacteria, Actinobacteria and Bacteroidetes, with Alphaproteobacteria, Gammaproteobacteria and Betaproteobacteria dominating at class-level taxonomy (Fig. 1). Interestingly, sand martin and common redstart FMs were dominated by the phylum Firmicutes and class Bacilli (Fig. 1), with the genera Streptococcus and Lactobacillus being most prevalent (Supplementary Fig. S2). A detailed summary of the FM taxonomic profiles of each passerine species is presented in Supplementary Fig. S2. 

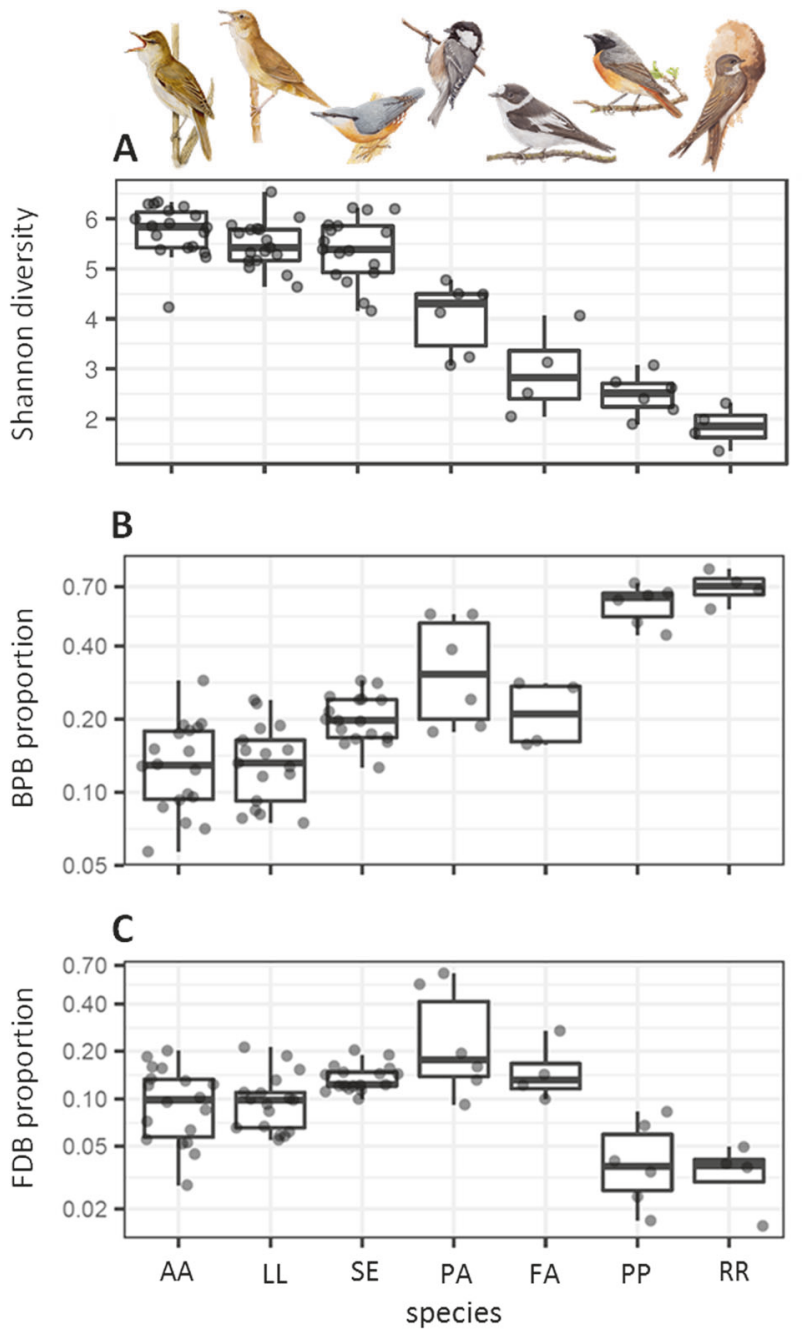

Fig. 2 Boxplots of (a) Shannon diversities, (b) BPB relative abundance and (c) keratinolytic FDB relative abundance within the feather microbiota of particular passerine host species (AA $=$ Acrocepalus arundinaceus, $\mathrm{LL}=$ Locustella luscinioides, $\mathrm{SE}=$ Sitta europaea, $\mathrm{PA}=$ Periparus ater, $\mathrm{FA}=$ Ficedula albicollis, $\mathrm{PP}=$ Phoenicurus phoenicurus, $\mathrm{RR}=$ Riparia riparia). (Illustrations by Jan Hošek)

\section{Feather microbiota richness and diversity}

FM community alpha diversity varied between individual host species (ANOVA: $F_{6,65}=60.51, p<0.0001, R^{2}=$ 0.85; Fig. 2a), with great reed warbler (Acrocephalus arundinaceus), Savi's warbler (Locustella luscinioides) and Eurasian nuthatch (Sitta europaea) having a more diverse FM than sand martin and common redstart (Fig. 2a).

NMDS ordination analysis for all dissimilarity indices and PERMANOVA analyses $\left(p<0.0001, R^{2}\right.$ range $=$ $0.35-0.42$ for all dissimilarity indices) confirmed pronounced interspecific variation in FM composition between host species (Fig. 3a-d). However, we also observed a strong correlation between geographic distance between sampling localities and community-wide dissimilarity in microbial profiles (Mantel tests: $p<0.0001, R^{2}$ range $=$ $0.38-0.66$, for all dissimilarity indices). Consequently, $\mathrm{db}$ RDA models were fitted to assess the extent to which the observed interspecific divergence in FM profiles was confounded by sampling different host species at different sites. The db-RDA analysis revealed significant effect of geography (i.e., sampling site locality) on all types of FM community dissimilarity, except for weighted UniFrac (Table 1). Despite this, interspecific differences in FM composition remained highly significant $(p<0.001)$ when controlling for the effect of geographic distance between sampling localities (Table 1). Three species from our dataset (reed warbler, Savi's warbler and collared flycatcher) were sampled from a single locality, however, whereas one species (sand martin) was sampled at multiple localities where data for other species were not collected (see Supplementary Table $\mathrm{S} 1$ for details). To demonstrate that the sampling scheme and associated db-RDA analyses did not confound the effect of species identity on FM divergence, an additional db-RDA analyses were conducted on a subset of the remaining three species. These analyses consistently supported a stronger effect of host species identity on FM divergence than geographic distance between sampling localities (Supplementary Table S3). Comparable results were obtained when we restricted these analyses to the three localities where we collected data for at least two different species simultaneously sampled at other localities, and when identity of sampling locality instead of geographic distances between sampling localities was treated as a factorial predictor (Supplementary Table S3).

In line with the db-RDA analysis, cluster heatmaps of the dominant bacterial OTUs showed host species identity to be a stronger predictor of FM divergence than sampling locality (Fig. 4). In particular, Eurasian nuthatch and coal tit (Periparus ater) FM profiles were grouped in single subclusters, despite being sampled at different sites, as were common redstarts and sand martins. In addition, hierarchical clustering was able to (almost) perfectly distinguish great reed and Savi's warblers originating from a single site. The collared flycatcher (Ficedula albicollis) was the only species that fell into two distinct sub-clusters (Fig. 4); however, this could not be ascribed to the effect of sample location as the species was only sampled at a single site.

\section{Effect of host species phylogeny on feather microbiota divergence}

In general, PACo analyses suggested co-divergence between FM composition and host phylogeny (PACo: $p<$ 0.0001 for all dissimilarity indices; Fig. 5a-d). Nevertheless, Procrustean superimposition plots suggested a relatively weak correlation for weighted UniFrac (Fig. 5d). Moreover, in line with the NMDS analysis, PACo revealed 

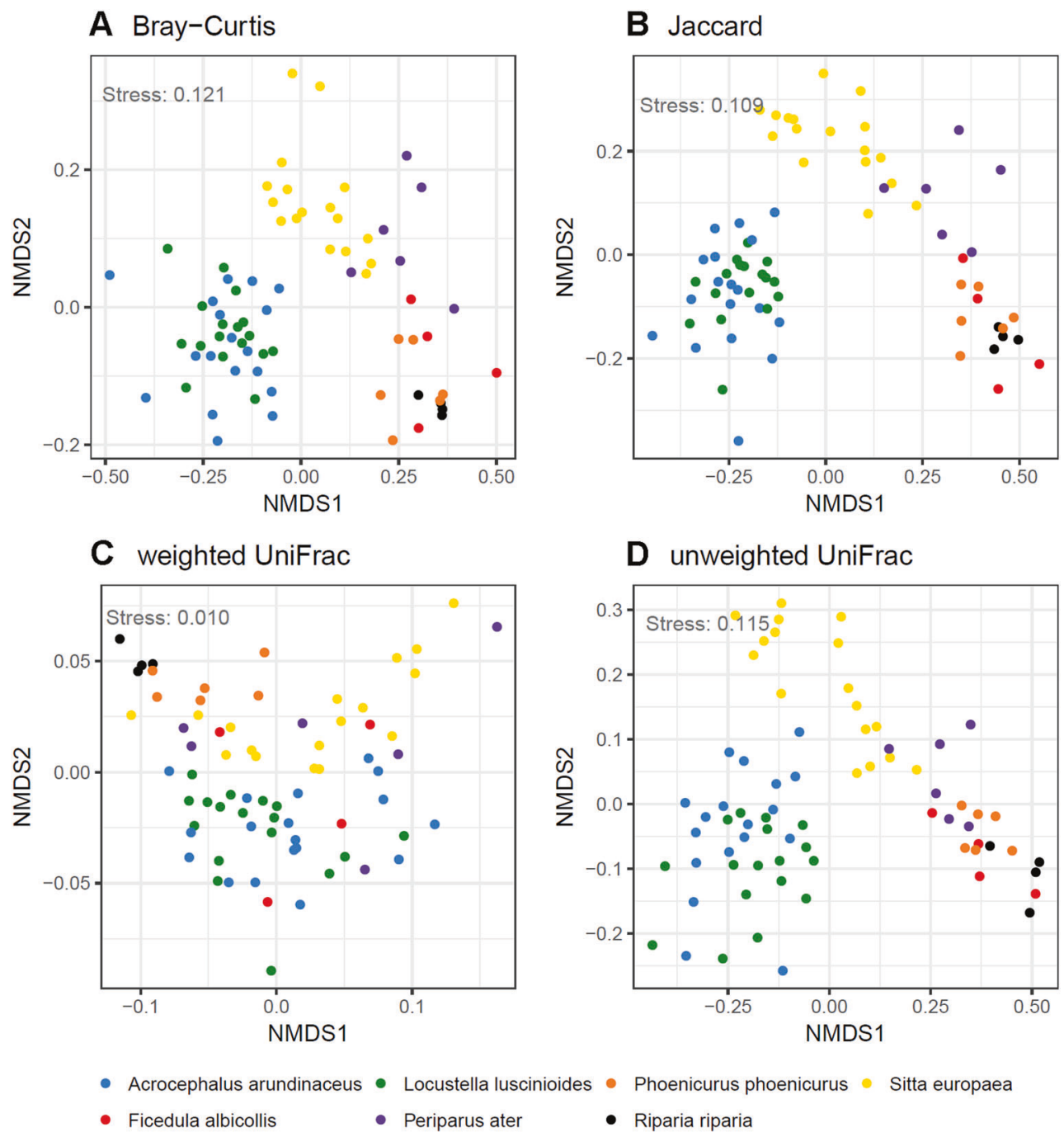

Fig. 3 Non-metric Multidimensional Scaling for among-sample divergence in composition ( $\beta$-diversity) of feather microbial community profiles based on (a) Bray-Curtis, (b) Jaccard, (c) weighted

UniFrac and (d) unweighted UniFrac dissimilarities. Different colours denote host species identity

convergence of two phylogenetically distant species, the common redstart and sand martin (Fig. 5a-c).

According to the db-RDA models, phylogeny explained from 5 to $12 \%$ of divergence in FM composition $(p<0.01$ for all dissimilarity indices) after controlling for FM geographic divergence. Depending on the type of FM dissimilarity indices, geography explained from 2 to $16 \%$ of FM divergence (Supplementary Fig. S3). All FM dissimilarity indices (except the unweighted UniFrac), ascribed around 5\% of FM divergence to the joint effect of phylogeny and geography.

\section{Proportion of BPB and keratinolytic FDB in feather microbiota}

Estimates of the FM fraction capable of producing different bacteriocin classes (i.e., Class I, II or III, see above for details) were highly correlated (Pearson correlation: $r>0.8, p<$
0.0001 for all pair-wise comparisons). The relative abundance of BPB and keratinolytic FDB estimated within all highquality reads was $24.5 \%$ and $12.2 \%$, respectively (Fig. 6). Moreover, $92 \%$ (i.e., $10.8 \%$ of the overall $12.2 \%$ ) of all estimated FDB was simultaneously assigned to BPB (Fig. 6). Furthermore, the BPB relative abundance within FM varied dramatically across species (ANOVA: $F_{6,65}=29.41, p<$ $0.0001, R^{2}=0.73$; Fig. $2 \mathrm{~b}$ ), as did the relative abundance of keratinolytic FDB (ANOVA: $F_{6,65}=12.46, p<0.0001, R^{2}=$ 0.53 ; Fig. 2c). Interestingly, sand martins and common redstarts harboured the highest BPB relative abundance (Fig. 2c), with the class Bacilli dominating in both (Fig. 1) and with the prevalence of Streptococcus spp. (53 and 45\%, respectively) and Lactobacillus spp. (9 and 7\%, respectively) (Supplementary Fig. S2). Sand martins and common redstarts also had the lowest estimated relative abundance (4\%) of keratinolytic FDB compared to other species (Fig. 2c). 
Table 1 Db-RDA ANOVA results for feather microbiota divergence associated with host species identity and geographic distance between sampling localities

Fig. 4 Heatmap denoting abundance of the dominant bacterial OTUs detected in the feather microbiota of individual passerine host species. Both rows and columns are clustered using the Ward algorithm. Identity of host species is indicated by column annotation, whereas row annotations correspond to the taxonomic assignation to the bacterial phyla

\begin{tabular}{llllrl}
\hline Dissimilarity index & Variable & df & Variance & \multicolumn{1}{l}{$F$} & $p$ \\
\hline w. UniFrac & Species & 6 & 0.0003 & 10.803 & 0.001 \\
& Between-locality geographic distance & 3 & 0.0000 & 0.282 & 0.945 \\
& Residual & 62 & 0.0002 & & \\
u. UniFrac & Species & 6 & 0.0230 & 43.999 & 0.001 \\
& Between-locality geographic distance & 3 & 0.0010 & 3.374 & 0.006 \\
& Residual & 62 & 0.0050 & & \\
Bray-Curtis & Species & 6 & 0.0390 & 75.383 & 0.001 \\
& Between-locality geographic distance & 3 & 0.0000 & 2.166 & 0.042 \\
& Residual & 62 & 0.0050 & & \\
Jaccard & Species & 6 & 0.0286 & 59.264 & 0.001 \\
& Between-locality geographic distance & 3 & 0.0000 & 2.938 & 0.01 \\
& Residual & 62 & 0.0050 & & \\
\hline
\end{tabular}

Tests were run on four types of dissimilarity index (weighted and unweighted UniFrac, Jaccard and Bray Curtis)

Variance associated with individual effect, values of $F$ statistics and marginal, premeditation-based $p$ values are shown

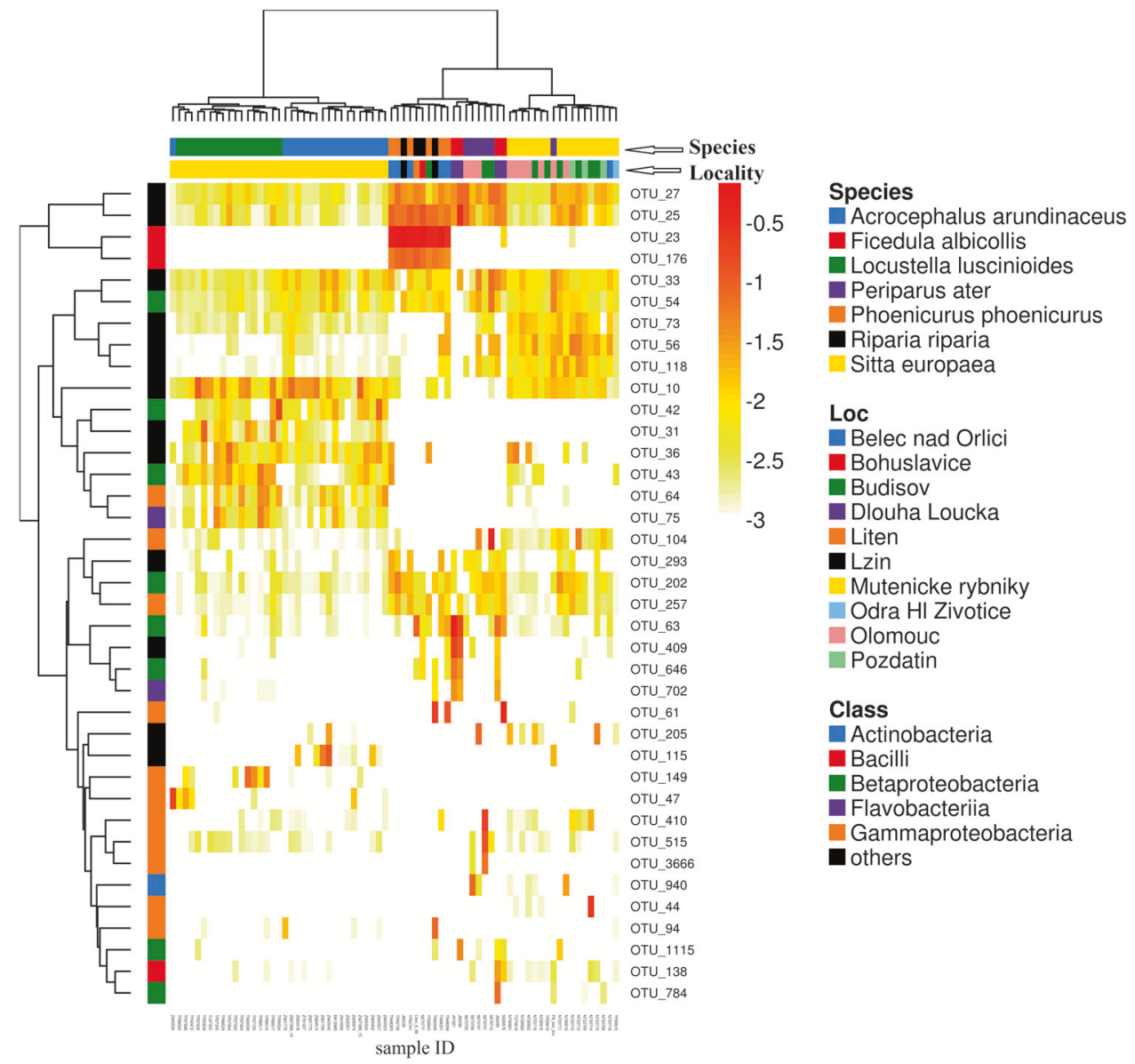

MCMCglmms revealed a negative effect of interspecific variation in BPB relative abundance on FM alpha diversity (MCMCglmm: slope $=-4.53,95 \% \mathrm{CrI}=-7.41,-1.54$; Fig. 7a). However, this relationship was not supported at the intraspecific level (slope $=-0.17,95 \% \mathrm{CrI}=-1.10,0.76$ ).
DIC was unaffected by presence $(\mathrm{DIC}=132.5)$ or absence $(\mathrm{DIC}=132.2)$ of species-specific effects (i.e., interaction between host species and intraspecific variability in BPB relative abundance) in the model, suggesting that the association between FM alpha diversity and intraspecific 


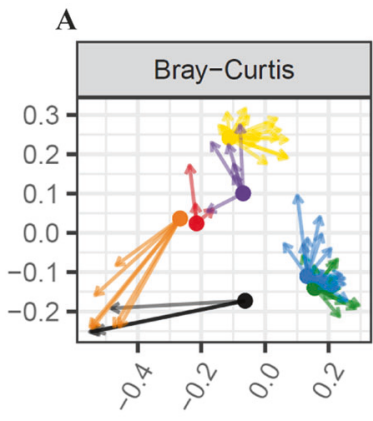

B

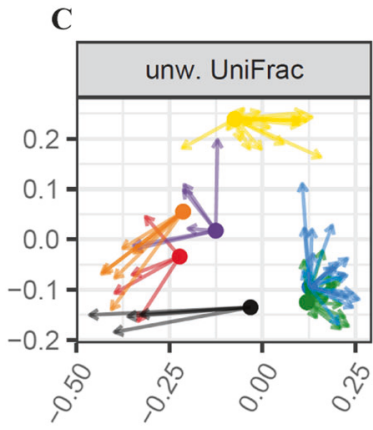

$\longrightarrow$ Acrocephalus arundinaceus

$\longrightarrow$ Ficedula albicollis

$\longrightarrow$ Locustella luscinioides

$\longrightarrow$ Periparus ater

Sitta europaea

Fig. 5 Procrustean superimposition for PCoA-scaled phylogenetic vs. feather microbiota distance. Feather microbiota divergence was calculated using (a) Bray-Curtis, (b) Jaccard, (c) unweighted UniFrac and (d) weighted UniFrac dissimilarities. Different colours denote host species identity

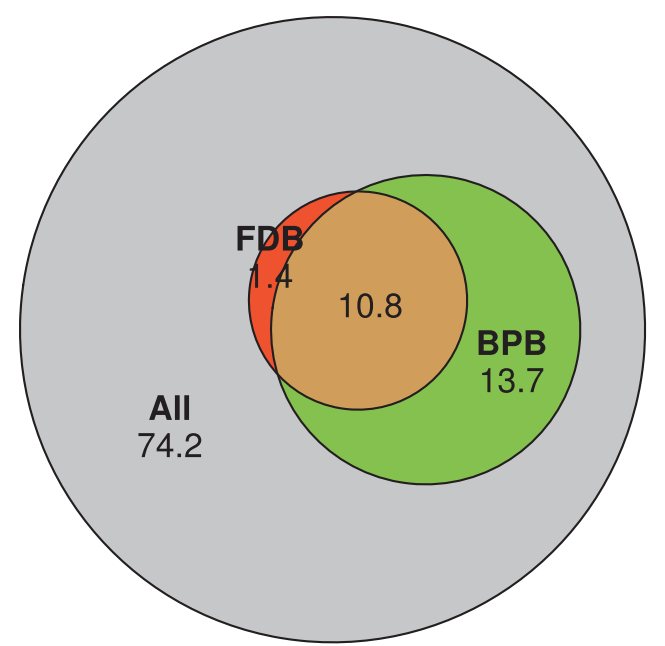

Fig. 6 Euler diagram for the relative abundance of bacteriocinproducing (BPB) and keratinolytic (FDB) OTUs within all observed OTUs in the feather microbiota of passerine host species. Numbers in the diagram denote the proportion (\%) of unique and shared OTUs

variability in BPB relative abundance did not differ between the host species.

In the case of keratinolytic FDB, we found a positive relationship between the relative abundance of FDB and
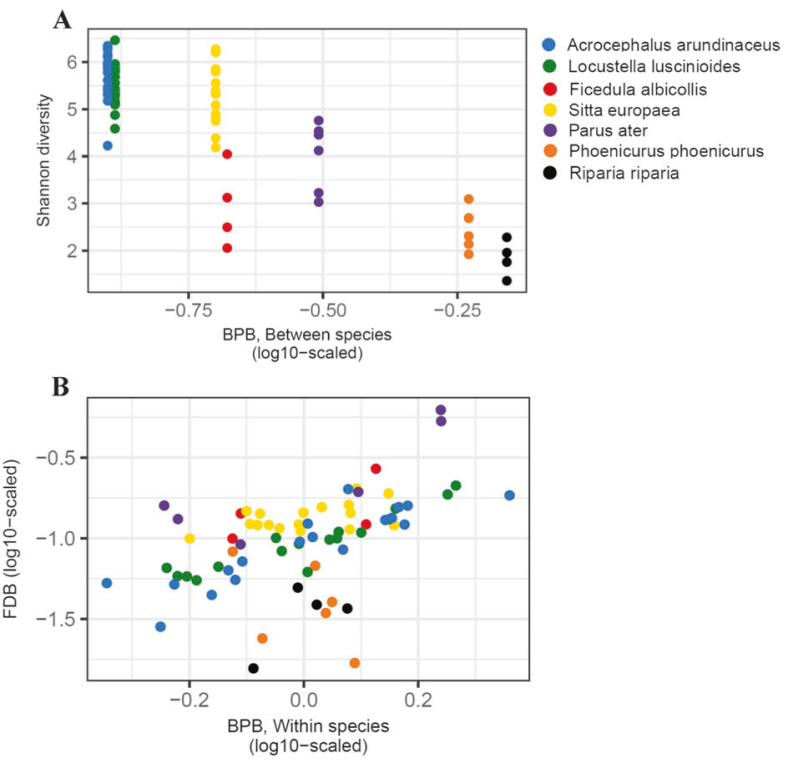

Fig. 7 Correlation between (a) BPB relative abundance and Shannon diversity and (b) relative abundance of BPB and keratinolytic FDB within the feather microbiota of host passerine species. Different colours denote host species identity

BPB within host species (MCMCglmm: slope $=1.01,95 \%$ $\mathrm{CrI}=0.78,1.24$; Fig. $7 \mathrm{~b}$ ); however, this relationship was not supported between host species (MCMCglmm: slope $=$ $-0.72,95 \% \mathrm{CrI}=-1.65,0.22$ ). A lower DIC for main effect models (DIC $=70.19)$ compared to models including interactions ( $\mathrm{DIC}=79.99)$ suggested a consistent association between BPB within species variation and FDB relative abundance between species.

We observed no association between FM alpha diversity and interspecific (MCMCglmm: slope $=2.62,95 \% \mathrm{CrI}=$ $-2.12,7.42$ ) or intraspecific (MCMCglmm: slope $=-0.29$, $95 \% \mathrm{CrI}=-0.96,0.39)$ variation in FDB relative abundance. Main effect models displayed a slightly lower DIC $(\mathrm{DIC}=130.0)$ than those with species-specific interaction $(\mathrm{DIC}=131.4)$, suggesting a lack of any pronounced relationship between host-specific FM diversity and variability in FDB relative abundance.

While $\mathrm{H}^{2}$ estimates were low $(<0.005$ for all models), associated posterior credible intervals were broad (ranging between $<0.0001$ and 0.8 in all models), implying that data from more species are required for reliable quantification of phylogenetic signal of these traits.

Finally, to explore if the existence of distinct FM phylotype affects associations between FM diversity and BPB and FDB relative abundances, we reran MCMCglmm simulations on a data subset where species whose FM was dominated by the genera Streptococcus and Lactobacillus (i.e., sand martin and common redstart) were excluded. Consistent with results for the whole dataset, we found a highly supported positive association between relative 
abundances of FDB and BPB within host species (MCMCglmm: slope $=1.06,95 \% \mathrm{CrI}=0.87,1.23)$ and no association between FM diversity and FDB relative abundance. Similarly, although significance was not further supported based on posteriori credible intervals in MCMCglmm for the data subset excluding species with specific FM phylotype, the slope of negative correlation between BPB relative abundance and FM alpha diversity at interspecific level remained almost unchanged (MCMCglmm: slope $=-4.73,95 \% \mathrm{CrI}=-17.79,8.68$ ).

\section{Discussion}

This study provides the first in-depth insights into interspecific diversity and the potential functional significance of FM in free-living passerine bird populations. In doing so, we revealed the underlying role of host species identity in shaping FM diversity and community profiles, with local environment having a weaker effect. Moreover, we observed a high prevalence of estimated BPB within passerine bird FM and pointed out their potential to be associated with species-specific FM diversity. These results suggest that avian feathers, as the most pronounced keratinous skin derivative, harbour species-specific bacterial communities that are likely to produce antimicrobials, thus serving as potentially important host guardians, similar to those on mammalian skin.

These findings are in agreement with the most recent comparative studies confirming that microbiota inhabiting vertebrate skin and its derivatives depend strongly on hostspecies ecology and environmental factors. Specifically, the interaction of host species identity and environmental conditions at both regional and local scales has been shown to shift diversity and composition of bat skin microbiota [72]. On the other hand, the skin microbiota of 89 tropical frog species sampled across 30 Madagascan sites was mostly determined by host-specific ecomorphology rather than sampling site characteristics [73], with identical outcomes also observed for skin microbial community profiles in temperate frogs [74]. In birds, three captive-bred finch species were shown to harbour highly species-specific skin microbiota, despite experiencing the same environments [75]. Similarly, the skin microbiota of a pelagic seabird, Leach's storm petrel (Oceanodroma leucorhoa), was shown to be independent of microbes from the surrounding environment and mainly affected by sex and individual genetic factors [15]. Finally, a recent study that also used $16 \mathrm{~S}$ rRNA profiling to compare the FMs of two sympatric lark species revealed horizontal acquisition of feather microbes from the shared environment [14]. Hence, it would appear that while the microbial assemblage of vertebrate integuments is driven to some extent by environmental niche-specific characteristics, host-specific factors have a stronger effect. At this time, however, the actual identity of those factors remains largely unknown in birds and most non-human vertebrates.

The FM of our focal passerine species was dominated by the phyla Proteobacteria, Actinobacteria, Bacteroidetes, Verrucomicrobia and Planctomycetes, with Alphaproteobacteria, Actinobacteria and Gammaproteobacteria dominant at class-level taxonomy. These results are consistent with a previous study investigating the FM of two sympatric lark species [14]. Our data revealed the existence of an FM phylotype distinct from FM profiles observed in most of our focal species, however, as well as those from previous studies [14, 76]. Interestingly, this phylotype was specific to sand martins and common redstarts, whose feathers were dominated by the phylum Firmicutes and the class Bacilli, with a high proportion of the genera Streptococcus and Lactobacillus. The genus Streptococcus is a common member of core human skin microbiota and represents a key commensals inhabiting the skin of healthy individuals [17]. Pathological increases in the abundance of streptococci are usually linked to skin damage caused by disease or immunocompromised individuals [77, 78]. In the present study, we can exclude pathological cutaneous infection as a cause of the high prevalence of streptococci in the feathers of these species as we sampled multiple individuals from geographically segregated populations. Furthermore, a recent study [79] analysing skin microbiota across mammals revealed a high abundance (16\%) of the genus Streptococcus in a chiropteran species, the Indian flying fox (Pteropus giganteus). Similarly, the family Streptococcaceae accounts for 3 to $24 \%$ of the total bacterial community on fish skin [80]. It would appear, therefore, that streptococci are common skin commensals in many free-living vertebrates, having a beneficial rather than pathogenic function for the host [17]. On the other hand, the genus Lactobacillus has only been identified as a member of the core skin microbiota of humans [81] and free-living primates [79] to date. Thus, the present study provides first evidence for the occurrence of these potentially beneficial symbiotic microorganisms inhabiting skin derivatives of non-mammalian vertebrates.

Our data suggest a pattern of phylosymbiosis for FMs in phylogenetically related species. Phylosymbiosis postulates that divergence in host-associated microbiota parallels host species phylogeny, which may indicate intensive coevolution between hosts and their microbiota [82]. However, phylosymbiosis at the community level does not always signify co-evolution. Recent studies have suggested that phylosymbiosis need not be conditioned by coevolutionary history but by environmental and hostspecific factors that may co-vary and contribute significantly to the microbiota assemblage [7, 82, 83]. This may lead to the absence, or an inability to detect, 
phylosymbiosis patterns in phylogenetically close species $[84,85]$ or, alternatively, it could result in a convergence of microbiota among unrelated species [86, 87], as observed in this study. We found highly convergent FMs in two phylogenetically distant species, both of which are often in close contact with the soil niche during breeding or foraging. Hence, we argue that, contrary to the strong phylosymbiosis observed for gut microbiota across vertebrate and invertebrate taxa $[12,82]$, convergence of external integument microbiota in free-living birds (or other vertebrates) is driven by both host-specific factors and, to some extent, by ecological niche sharing, as postulated by the "niche-driven microbiota assembly hypothesis' [14]. Clearly, more studies investigating the factors responsible for shaping and maintaining functional microbiota living on the skin and its derivatives in non-human vertebrates are needed.

Our bioinformatic estimates suggest that all the bird species studied hosted both BPB and FDB in their feathers. In comparison, a previous culture-based study investigating FDB across 154 avian species recorded just $39 \%$ of all birds analysed as having FDB in their feathers [25]. We assume that the difference between the two studies may have resulted from the fact that only specific FDB were investigated in the latter study, meaning that the results of the two studies cannot be compared. Moreover, it is worth noting that the use of culture-based techniques commonly leads to underestimation of microbial abundance and false negative results [88]. Of more interest, however, is the discovery of a potentially unique role for BPB within feather microbial communities. We found that relative abundance of estimated BPB was negatively associated with FM alpha diversity in all the passerines studied. This effect was most pronounced in common redstarts and sand martins, whose feather microbial communities were dominated by BPB of the genera Streptococcus and Lactobacillus, resulting in the lowest FM alpha diversity of all the species studied. As most species within the genera Streptococcus and Lactobacillus have been shown to produce potent bacteriocins $[39,58,89,90]$, their estimated dominance in the feathers of two focal species suggests a strong potential to shape their feather microbial profiles. On the other hand, great reed and Savi's warblers had the lowest BPB relative abundances of all focal species, but the highest FM alpha diversities. This is in accordance with previous studies documenting a plethora of BPB within the gut microbiota of vertebrates $[91,92]$ that are able to significantly augment niche competition $[42,43]$ and decrease gut microbiota diversity and composition [90, 93]. A few studies have also recorded the presence of resident commensals, including bacteriocinproducing streptococci, as underlying agents maintaining a balanced and healthy human skin microbiota [17]. Moreover, experimental evidence exists for the importance of skin surface symbionts able to switch skin microbiota abundance and diversity in tadpoles [94]. Though evidence exists for the potential of mechanisms based on preening and preen gland secretions to affect FM [16, 36, 95], the present study is the first to point out an association between antimicrobial compound-producing bacterial commensals and FM divergence in free-living birds. However, experimental studies are needed to verify the likely potential of BPB to substantially shape FM. In addition, we documented that after the exclusion of the species that harboured microbial phylotype dominated by the genera Streptococcus and Lactobacillus from the dataset, regression slope of negative correlation between FM diversity and BPB relative abundance was almost identical, yet its significance was not further supported. Consequently, we cannot unambiguously resolve, whether such decrease of support for the association between BPB relative abundance vs FM alpha diversity was caused by low statistical power or by the effect FM phylotype. More robust comparative datasets are therefore needed to resolve to what extent existence of contrasting FM phylotypes is involved in the negative association between BPB relative abundance and FM alpha diversity.

Recent studies have documented skin microbial commensals protecting the skin against pathogens and detrimental microorganisms in humans [96], amphibians $[21,22,97]$ and fish [80]. While there was no negative correlation between BPB and FDB in this study, we observed a positive association between $\mathrm{BPB}$ relative abundance and FDB at the intraspecific level. As such, our results differ from those of the most recent research on humans and non-human vertebrate skin microbiota. In the case of potentially harmful FDB, however, it is worth noting that only one in vivo study has provided evidence for a negative effect on feather quality [98], while two other studies showed no relationship between FDB and feather wear $[34,35]$. These findings partially support our speculation on a positive association between BPB and FDB because the fact that most FDB (mostly from the genera Bacillus and Pseudomonas) are simultaneously BPB. As such, they are able to produce bacteriocins $[99,100]$ that may augment niche-competition within FMs and maintain well-balanced feather commensal microbiota. We are aware that our findings are limited to BPB and FDB relative abundance within $\mathrm{FM}$, which precludes an evaluation of any relationship between specific BPB and FDB absolute abundance and vice versa. Nevertheless, our findings suggest that the potentially deleterious role of FDB in birds should be re-evaluated and the occurrence of FDB on feathers should not necessarily be interpreted as detrimental, but at least neutral or even beneficial for birds.

For the first time, this study provides support for the hypothesis that feathers, as the most pronounced keratinous skin derivative, harbour species-specific bacterial communities that are able to produce antimicrobials similar to those on 
mammalian skin. While the positive effect of skin microbiota on host health and immune status has previously only been well documented in humans, the present study significantly extends our knowledge of the functional potential of microbiota inhabiting skin derivatives and outlines avenues for future research on the driving forces behind the evolution of host-integument microbiomes in non-human vertebrates.

\section{Data availability}

All sequence data are deposited in the form of fastq files for each sample in the European Nucleotide Archive (ENA). Metadata for these files and their accession numbers are provided in Supplementary Table S4.

Acknowledgements Many thanks go to Lucie Schmiedová and Eva Holánová for their help with laboratory work, Radka Valterová for sample sorting and processing, Jan Hošek for providing us with illustrations, Jaroslav Koleček, Radka Reifová and Michaela Krestová for their help with permit administration and Kevin Roche for English proofreading and editing the manuscript. We are grateful for access to computing and storage facilities owned by parties and projects contributing to the National Grid Infrastructure MetaCentrum provided under the programme 'Projects of Large Research, Development, and Innovations Infrastructures' (CESNET LM2015042).

Funding This study was fully supported by Czech Science Foundation (CSF) Grant 14-16861P. JK was partially supported by Charles University Grant Agency Project 1438417 . VGJ was partially supported by Institutional Support for Young Talented Researchers of the Czech Academy of Sciences RVO: 68081766.

Author contributions VGJ developed the study concept and experimental design, supervised the project, performed laboratory work and drafted the manuscript. JK conducted complete bioinformatics and data analysis. VGJ, PP, MP, PA, VB, KŠ, PH and JP collected the samples. JK, PP, MP, PA, VB, KŠ and PH contributed to the manuscript preparation.

\section{Compliance with ethical standards}

Conflict of interest The authors declare that they have no conflict of interest.

Publisher's note: Springer Nature remains neutral with regard to jurisdictional claims in published maps and institutional affiliations.

Open Access This article is licensed under a Creative Commons Attribution 4.0 International License, which permits use, sharing, adaptation, distribution and reproduction in any medium or format, as long as you give appropriate credit to the original author(s) and the source, provide a link to the Creative Commons license, and indicate if changes were made. The images or other third party material in this article are included in the article's Creative Commons license, unless indicated otherwise in a credit line to the material. If material is not included in the article's Creative Commons license and your intended use is not permitted by statutory regulation or exceeds the permitted use, you will need to obtain permission directly from the copyright holder. To view a copy of this license, visit http://creativecommons. org/licenses/by/4.0/.

\section{References}

1. Fromont C, Riegler M, Cook JM. Relative abundance and strain diversity in the bacterial endosymbiont community of a sapfeeding insect across its native and introduced geographic range. Microb Ecol. 2017;74:722-34.

2. Groussin M, Mazel F, Sanders JG, Smillie CS, Lavergne S, Thuiller $\mathrm{W}$, et al. Unraveling the processes shaping mammalian gut microbiomes over evolutionary time. Nat Commun. 2017;8:12.

3. Clavel T, Gomes-Neto JC, Lagkouvardos I, Ramer-Tait AE. Deciphering interactions between the gut microbiota and the immune system via microbial cultivation and minimal microbiomes. Immunol Rev. 2017;279:8-22.

4. Kundu P, Blacher E, Elinav E, Pettersson S. Our gut microbiome: the evolving inner self. Cell. 2017;171:1481-93.

5. Moeller AH, Suzuki TA, Lin D, Lacey EA, Wasser SK, Nachman MW. Dispersal limitation promotes the diversification of the mammalian gut microbiota. $P$ Natl Acad Sci USA. 2017;114:13768-73.

6. Ren TT, Boutin S, Humphries MM, Dantzer B, Gorrell JC, Coltman DW, et al. Seasonal, spatial, and maternal effects on gut microbiome in wild red squirrels. Microbiome. 2017;5:163.

7. Carrier TJ, Reitzel AM. The hologenime across environments and the implications of a host-associated microbial repertoire. Front Microbiol. 2017;8:13.

8. Colston TJ, Jackson CR. Microbiome evolution along divergent branches of the vertebrate tree of life: what is known and unknown. Mol Ecol. 2016;25:3776-800.

9. Hubert J, Erban T, Kopecky J, Sopko B, Nesvorna M, Lichovnikova $\mathrm{M}$, et al. Comparison of microbiomes between red poultry mite populations (Dermanyssus gallinae): predominance of bartonella-like bacteria. Microb Ecol. 2017;74: 947-60.

10. Wilkinson TJ, Cowan AA, Vallin HE, Onime LA, Oyama LB, Cameron SJ, et al. Characterization of the microbiome along the gastrointestinal tract of growing Turkeys. Front Microbiol. 2017;8:1-11.

11. Roggenbuck M, Schnell IB, Blom N, Baelum J, Bertelsen MF, Ponten TS, et al. The microbiome of New World vultures. Nat Commun. 2014;5:5498.

12. Kropackova L, Tesicky M, Albrecht T, Kubovciak J, Cizkova D, Tomasek $\mathrm{O}$, et al. Codiversification of gastrointestinal microbiota and phylogeny in passerines is not explained by ecological divergence. Mol Ecol. 2017;26:5292-304.

13. Kreisinger J, Cizkova D, Kropackova L, Albrecht T. Cloacal microbiome structure in a long-distance migratory bird assessed using deep 16sRNA pyrosequencing. PLoS ONE. 2015;10: $\mathrm{e} 0137401$.

14. van Veelen HPJ, Salles JF, Tieleman BI. Multi-level comparisons of cloacal, skin, feather and nest-associated microbiota suggest considerable influence of horizontal acquisition on the microbiota assembly of sympatric woodlarks and skylarks. Microbiome. 2017;5:156.

15. Pearce DS, Hoover BA, Jennings S, Nevitt GA, Docherty KM. Morphological and genetic factors shape the microbiome of a seabird species (Oceanodroma leucorhoa) more than environmental and social factors. Microbiome. 2017;5:146.

16. Jacob S, Salle L, Zinger L, Chaine AS, Ducamp C, Boutault L, et al. Chemical regulation of body feather microbiota in a wild bird. Mol Ecol. 2018;27:1727-38.

17. Barnard E, Li HY. Shaping of cutaneous function by encounters with commensals. J Physiol-London. 2017;595:437-50.

18. Cheng YY, Belov K. Antimicrobial protection of marsupial pouch young. Front Microbiol. 2017;8:8. 
19. Chehoud C, Rafail S, Tyldsley AS, Seykora JT, Lambris JD, Grice EA. Complement modulates the cutaneous microbiome and inflammatory milieu. $P$ Natl Acad Sci USA. 2013;110:15061-6.

20. Meisel JS, Sfyroera G, Bartow-McKenney C, Gimblet C, Bugayev J, Horwinski J, et al. Commensal microbiota modulate gene expression in the skin. Microbiome. 2018;6:15.

21. Kueneman JG, Weiss S, McKenzie VJ. Composition of microeukaryotes on the skin of the cascades Frog (Rana cascadae) and patterns of correlation between skin microbes and batrachochytrium dendrobatidis. Front Microbiol. 2017;8:2350.

22. Barnhart K, Forman ME, Umile TP, Kueneman J, McKenzie V, Salinas I, et al. Identification of Bufadienolides from the Boreal Toad, Anaxyrus boreas, active against a fungal pathogen. Microb Ecol. 2017;74:990-1000.

23. Xu X, Zhou ZH, Dudley R, Mackem S, Chuong CM, Erickson $\mathrm{GM}$, et al. An integrative approach to understanding bird origins. Science. 2014;346:1341-8.

24. Fulop A, Czirjak GA, Pap PL, Vagasi CI. Feather-degrading bacteria, uropygial gland size and feather quality in House Sparrows Passer domesticus. Ibis. 2016;158:362-70.

25. Kent CM, Burtt EH. Feather-degrading bacilli in the plumage of wild birds: prevalence and relation to feather wear. Auk. 2016;133:583-92.

26. Leclaire S, Czirjak GA, Hammouda A, Gasparini J. Feather bacterial load shapes the trade-off between preening and immunity in pigeons. BMC Evol Biol. 2015;15:8.

27. Sharma R, Devi S. Versatility and commercial status of microbial keratinases: a review. Rev Environ Sci Bio. 2018;17:19-45.

28. Gupta R, Ramnani P. Microbial keratinases and their prospective applications: an overview. Appl Microbiol Biot. 2006;70:21-33.

29. Goldstein G, Flory KR, Browne BA, Majid S, Ichida JM, Burtt EH. Bacterial degradation of black and white feathers. Auk. 2004;121:656-9.

30. Gunderson AR, Frame AM, Swaddle JP, Forsyth MH. Resistance of melanized feathers to bacterial degradation: is it really so black and white? J Avian Biol. 2008;39:539-45.

31. Ruiz-De-Castaneda R, Burtt EH, Gonzalez-Braojos S, Moreno J. Bacterial degradability of an intrafeather unmelanized ornament: a role for feather-degrading bacteria in sexual selection? Biol $\mathrm{J}$ Linn Soc. 2012;105:409-19.

32. Gunderson AR, Forsyth MH, Swaddle JP. Evidence that plumage bacteria influence feather coloration and body condition of eastern bluebirds Sialia sialis. J Avian Biol. 2009;40:440-7.

33. Shawkey MD, Pillai SR, Hill GE, Siefferman LM, Roberts SR. Bacteria as an agent for change in structural plumage color: correlational and experimental evidence. Am Nat. 2007;169: S112-S21.

34. Cristol DA, Armstrong JL, Whitaker JM, Forsyth MH. Featherdegrading bacteria do not affect feathers on captive birds. Auk. 2005; 122:222-30.

35. Jacob S, Colmas L, Parthuisot N, Heeb P. Do feather-degrading bacteria actually degrade feather colour? No significant effects of plumage microbiome modifications on feather colouration in wild great tits. Naturwissenschaften. 2014;101:929-38.

36. Braun MS, Sporer F, Zimmermann S, Wink M. Birds, featherdegrading bacteria and preen glands: the antimicrobial activity of preen gland secretions from turkeys (Meleagris gallopavo) is amplified by keratinase. FEMS Microbiol Ecol. 2018;94:1-15.

37. Stein T. Bacillus subtilis antibiotics: structures, syntheses and specific functions. Mol Microbiol. 2005;56:845-57.

38. Riley MA, Wertz JE. Bacteriocins: evolution, ecology, and application. Annu Rev Microbiol. 2002;56:117-37.

39. Chao L, Levin BR. Structured habitats and the evolution of anticompetitor toxins in bacteria. P Natl Acad Sci-Biol. 1981;78:6324-8.
40. Dobson A, Cotter PD, Ross RP, Hill C. Bacteriocin production: a probiotic trait? Appl Environ Microb. 2012;78:1-6.

41. Cotter PD, Ross RP, Hill C. Bacteriocins-a viable alternative to antibiotics? Nat Rev Microbiol. 2013;11:95-105.

42. Drissi F, Buffet S, Raoult D, Merhej V. Common occurrence of antibacterial agents in human intestinal microbiota. Front Microbiol. 2015;6:441.

43. Kommineni S, Bretl DJ, Lam V, Chakraborty R, Hayward M, Simpson $\mathrm{P}$, et al. Bacteriocin production augments niche competition by enterococci in the mammalian gastrointestinal tract. Nature. 2015;526:719-22.

44. Martin-Platero AM, Valdivia E, Ruiz-Rodriguez M, Soler JJ, Martin-Vivaldi M, Maqueda M, et al. Characterization of antimicrobial substances produced by Enterococcus faecalis MRR 10-3, isolated from the uropygial gland of the hoopoe (Upupa epops). Appl Environ Microbiol. 2006;72:4245-9.

45. Martin-Vivaldi M, Pena A, Peralta-Sanchez JM, Sanchez L, Ananou S, Ruiz-Rodriguez M, et al. Antimicrobial chemicals in hoopoe preen secretions are produced by symbiotic bacteria. Proc R Soc B-Biol Sci. 2010;277:123-30.

46. Ruiz-Rodriguez M, Martinez-Bueno M, Martin-Vivaldi M, Valdivia E, Soler JJ. Bacteriocins with a broader antimicrobial spectrum prevail in enterococcal symbionts isolated from the hoopoe's uropygial gland. FEMS Microbiol Ecol. 2013;85:495-502.

47. Martinez-Garcia A, Soler JJ, Rodriguez-Ruano SM, MartinezBueno M, Martin-Platero AM, Juarez-Garcia N, et al. Preening as a Vehicle for Key Bacteria in Hoopoes. Microb Ecol. 2015;70:1024-33.

48. Kreisinger J, Kropackova L, Petrzelkova A, Adamkova M, Tomasek O, Martin JF, et al. Temporal stability and the effect of transgenerational transfer on fecal microbiota structure in a long distance migratory bird. Front Microbiol. 2017;8:50.

49. Klindworth A, Pruesse E, Schweer T, Peplies J, Quast C, Horn $\mathrm{M}$, et al. Evaluation of general 16S ribosomal RNA gene PCR primers for classical and next-generation sequencing-based diversity studies. Nucleic Acids Res. 2013;41:e1.

50. Zhang JJ, Kobert K, Flouri T, Stamatakis A. PEAR: a fast and accurate illumina paired-end reAd mergeR. Bioinformatics. 2014;30:614-20.

51. Jiang HS, Lei R, Ding SW, Zhu SF. Skewer: a fast and accurate adapter trimmer for next-generation sequencing paired-end reads. BMC Bioinformatics. 2014;15:182.

52. Callahan BJ, McMurdie PJ, Rosen MJ, Han AW, Johnson AJA, Holmes SP. DADA2: High-resolution sample inference from Illumina amplicon data. Nat Methods. 2016;13:581-90.

53. Edgar RC, Haas BJ, Clemente JC, Quince C, Knight R. UCHIME improves sensitivity and speed of chimera detection. Bioinformatics. 2011;27:2194-200.

54. DeSantis TZ, Hugenholtz P, Larsen N, Rojas M, Brodie EL, Keller K, et al. Greengenes, a chimera-checked 16S rRNA gene database and workbench compatible with ARB. Appl Environ Microbiol. 2006;72:5069-72.

55. Caporaso JG, Bittinger K, Bushman FD, DeSantis TZ, Andersen GL, Knight R. PyNAST: a flexible tool for aligning sequences to a template alignment. Bioinformatics. 2010;26:266-7.

56. Price MN, Dehal PS, Arkin AP. FastTree: computing large minimum evolution trees with profiles instead of a distance matrix. Mol Biol Evol. 2009;26:1641-50.

57. McMurdie PJ, Holmes S. phyloseq: an R package for reproducible interactive analysis and graphics of microbiome census data. PLoS ONE. 2013;8:e61217.

58. van Heel AJ, de Jong A, Montalban-Lopez M, Kok J, Kuipers OP. BAGEL3: automated identification of genes encoding bacteriocins and (non-)bactericidal posttranslationally modified peptides. Nucleic Acids Res. 2013;41(W1):W448-W53. 
59. Quast C, Pruesse E, Yilmaz P, Gerken J, Schweer T, Yarza P, et al. The SILVA ribosomal RNA gene database project: improved data processing and web-based tools. Nucleic Acids Res. 2013;41(D1):D590-D6.

60. Edgar RC. Search and clustering orders of magnitude faster than BLAST. Bioinformatics. 2010;26:2460-1.

61. Chikindas ML, Weeks R, Drider D, Chistyakov VA, Dicks LMT. Functions and emerging applications of bacteriocins. Curr Opin Biotech. 2018;49:23-8.

62. Lozupone C, Knight R. UniFrac: a new phylogenetic method for comparing microbial communities. Appl Environ Microbiol. 2005;71:8228-35.

63. Borcard D, Legendre P. All-scale spatial analysis of ecological data by means of principal coordinates of neighbour matrices. Ecol Model. 2002;153:51-68.

64. Gaujoux R, Seoighe C. A flexible R package for nonnegative matrix factorization. BMC Bioinformatics. 2010;11:367.

65. Balbuena JA, Miguez-Lozano R, Blasco-Costa I. PACo: a novel procrustes application to cophylogenetic analysis. PLoS ONE. 2013;8:e61048.

66. Jetz W, Thomas GH, Joy JB, Hartmann K, Mooers AO. The global diversity of birds in space and time. Nature. 2012;491:444-8.

67. Hadfield JD. MCMC methods for multi-response generalized linear mixed models: the MCMCglmm R package. J Stat Softw. 2010;33:1-22.

68. van de Pol MV, Wright J. A simple method for distinguishing within- versus between-subject effects using mixed models. Anim Behav. 2009;77:753-8.

69. Guillerme T, Healy K. mulTree: a package for running MCMCglmm analysis on multiple trees. ZENODO. 2014. https://doi.org/10.5281/zenodo.12902.

70. Pagel M. Inferring the historical patterns of biological evolution. Nature. 1999;401:877-84.

71. Garamszegi LZ (eds). Modern phylogenetic comparative methods and their application in evolutionary biology: concepts and practice, 1st ed. Heidelberg, DE: Springer; 2014.

72. Avena CV, Parfrey LW, Leff JW, Archer HM, Frick WF, Langwig KE, et al. Deconstructing the bat skin microbiome: influences of the host and the environment. Front Microbiol. 2016;7:1753.

73. Bletz MC, Archer H, Harris RN, McKenzie VJ, Rabemananjara FCE, Rakotoarison A, et al. Host ecology rather than host phylogeny drives amphibian skin microbial community structure in the biodiversity hotspot of Madagascar. Front Microbiol. 2017;8:1-14.

74. Kueneman JG, Parfrey LW, Woodhams DC, Archer HM, Knight $\mathrm{R}$, McKenzie VJ. The amphibian skin-associated microbiome across species, space and life history stages. Mol Ecol. 2014;23:1238-50.

75. Engel K, Sauer J, Jünemann SJ, Winkler A, Wibberg D, Kalinowski J, et al. Individual and species-specific skin microbiomes in three different Estrildid Finch species revealed by $16 \mathrm{~S}$ amplicon sequencing. Microb Ecol. 2018;76:518-29.

76. Shawkey MD, Hussain MJ, Strong AL, Hagelin JC, Vollmer AC, Hill GE. Use of culture-independent methods to compare bacterial assemblages on feathers of crested and least Auklets (Aethia cristatella and Aethia pusilla) with those of passerines. Waterbirds. 2006;29:507-11.

77. Tomi NS, Kranke B, Aberer E. Staphylococcal toxins in patients with psoriasis, atopic dermatitis, and erythroderma, and in healthy control subjects. J Am Acad Dermatol. 2005;53:67-72.

78. Gao Z, Tseng CH, Strober BE, Pei ZH, Blaser MJ. Substantial alterations of the cutaneous bacterial biota in psoriatic lesions. PLoS ONE. 2008;3:e2719.
79. Ross AA, Muller K, Weese S, Neufeld J. Comprehensive skin microbiome analysis reveals the uniqueness of human-associated microbial communities among the class Mammalia. bioRxiv. 2018. https://doi.org/10.1101/201434.

80. Lowrey L, Woodhams DC, Tacchi L, Salinas I. Topographical mapping of the rainbow trout (Oncorhynchus mykiss) microbiome reveals a diverse bacterial community with antifungal properties in the skin. Appl Environ Microbiol. 2015;81:6915-25.

81. Li X, Yuan C, Xing LC, Humbert P. Topographical diversity of common skin microflora and its association with skin environment type: an observational study in Chinese women. Sci RepUK. 2017;7:18046.

82. Brooks AW, Kohl KD, Brucker RM, van Opstal EJ, Bordenstein SR. Phylosymbiosis: relationships and functional effects of microbial communities across host evolutionary history. PLoS Biol. 2016;14:e2000225.

83. Hird SM, Sanchez C, Carstens BC, Brumfield RT. Comparative gut microbiota of 59 neotropical bird species. Front Microbiol. 2015;6:1403.

84. Baxter NT, Wan JJ, Schubert AM, Jenior ML, Myers P, Schloss $\mathrm{PD}$. Intra and interindividual variations mask interspecies variation in the microbiota of sympatric peromyscus populations. Appl Environ Microbiol. 2015;81:396-404.

85. Schmitt S, Tsai P, Bell J, Fromont J, Ilan M, Lindquist N, et al. Assessing the complex sponge microbiota: core, variable and species-specific bacterial communities in marine sponges. ISME J. 2012;6:564-76.

86. Carrier TJ, Reitzel AM. Convergent shifts in host-associated microbial communities across environmentally elicited phenotypes. Nat Commun. 2018;9:9.

87. Delsuc F, Metcalf JL, Parfrey LW, Song SJ, Gonzalez A, Knight R. Convergence of gut microbiomes in myrmecophagous mammals. Mol Ecol. 2014;23:1301-17.

88. Shawkey MD, Mills KL, Dale C, Hill GE. Microbial diversity of wild bird feathers revealed through culture-based and cultureindependent techniques. Microb Ecol. 2005;50:40-7.

89. Riley MA, Wertz JE. Bacteriocin diversity: ecological and evolutionary perspectives. Biochimie. 2002;84:357-64.

90. Collins FWJ, O'Connor PM, O'Sullivan O, Gomez-Sala B, Rea $\mathrm{MC}$, Hill $\mathrm{C}$, et al. Bacteriocin gene-trait matching across the complete lactobacillus Pan-genome. Sci Rep-UK. 2017; $7: 14$.

91. Walsh CJ, Guinane CM, Hill C, Ross RP, O'Toole PW, Cotter PD. In silico identification of bacteriocin gene clusters in the gastrointestinal tract, based on the Human Microbiome Project's reference genome database. BMC Microbiol. 2015; 15:183.

92. Zheng JS, Ganzle MG, Lin XXB, Ruan LF, Sun M. Diversity and dynamics of bacteriocins from human microbiome. Environ Microbiol. 2015;17:2133-43.

93. Umu OCO, Bauerl C, Oostindjer M, Pope PB, Hernandez PE, Perez-Martinez G, et al. The potential of class II bacteriocins to modify gut microbiota to improve host health. PLoS ONE. 2016;11:10.

94. Davis LR, Bigler L, Woodhams DC. Developmental trajectories of amphibian microbiota: response to bacterial therapy depends on initial community structure. Environ Microbiol. 2017;19:1502-17.

95. Moreno-Rueda G. Preen oil and bird fitness: a critical review of the evidence. Biol Rev. 2017;92:2131-43.

96. Nakatsuji T, Chen TH, Narala S, Chun KA, Two AM, Yun T, et al. Antimicrobials from human skin commensal bacteria protect against Staphylococcus aureus and are deficient in atopic dermatitis. Sci Transl Med. 2017;9:eaah4680. 
97. Rebollar EA, Hughey MC, Medina D, Harris RN, Ibanez R, Belden LK. Skin bacterial diversity of Panamanian frogs is associated with host susceptibility and presence of Batrachochytrium dendrobatidis. ISME J. 2016;10:1682-95.

98. Leclaire S, Pierret P, Chatelain M, Gasparini J. Feather bacterial load affects plumage condition, iridescent color, and investment in preening in pigeons. Behav Ecol. 2014;25:1192-8.
99. Ghequire MGK, Kemland L, Anoz-Carbonell E, Buchanan SK, De Mot R. A natural chimeric pseudomonas bacteriocin with novel pore-forming activity parasitizes the ferrichrome transporter. mBio. 2017;8:12.

100. Lim KB, Balolong MP, Kim SH, Oh JK, Lee JY, Kang DK. Isolation and characterization of a broad spectrum bacteriocin from Bacillus amyloliquefaciens RX7. Biomed Res Int. 2016. https://doi.org/10.1155/2016/8521476. 